\title{
The role of contaminants in the variation of adhesion, friction, and electrical conduction properties of carbide-coated scanning probe tips and $\mathrm{Pt}(111)$ in ultrahigh vacuum
}

\author{
M. Enachescu \\ Materials Sciences Division, Lawrence Berkeley National Laboratory, University of California, Berkeley, \\ California 94720 \\ R. W. Carpick \\ Engineering Physics Department, University of Wisconsin-Madison, Madison, Wisconsin 53706 \\ D. F. Ogletree and M. Salmeron ${ }^{\text {a) }}$ \\ Materials Sciences Division, Lawrence Berkeley National Laboratory, University of California, Berkeley, \\ California 94720
}

(Received 8 December 2003; accepted 18 March 2004)

\begin{abstract}
Scanning probe microscopy was used to investigate the tribological properties of nanoscale tips in contact with a Pt(111) single-crystal surface under ultrahigh vacuum conditions. The tips were coated with a tungsten carbide film, which contained a significant fraction of oxygen. The electrically conductive tip made it possible to alternate between contact measurements and noncontact scanning tunneling microscopy. Several types of interfaces were found depending on the chemical state of the surfaces. The first type is characterized by strong irreversible adhesion followed by material transfer between tip and sample. Low adhesion and no material transfer characterize a second type of contact, which are associated with the presence of passivating adsorbates in both (full passivation) or in one of the two contacting surfaces (half-passivation). Half-passivated contacts in which the clean side is the $\mathrm{Pt}(111)$ sample gave rise to periodic stick-slip friction behavior with a period equal to the atomic lattice constant of the Pt(111) surface. Local electrical conductivity measurements show a clear correlation between electronic and friction properties, with ohmic behavior on clean regions of the Pt surface and semiconductor-like behavior on areas covered with adsorbates. (C) 2004 American Institute of Physics.
\end{abstract}

[DOI: $10.1063 / 1.1738536$ ]

\section{INTRODUCTION}

The presence of even minute amounts of adsorbates on the surfaces of contacting bodies can have a striking influence on their tribological properties. Strong adhesion, characteristic of chemically active surfaces, can lead to rupture of the contact by failure within the bulk of the bodies, which often results in material transferred from one to the other, or left as debris in or near the contact zone. This material, sometimes referred to as "the third body" or a "transfer film," depending on the extent to which it remains bonded to one of the surfaces, influences the transient behavior of the sliding contact and can completely dominate the steady-state sliding behavior of many interfaces, especially for lowfriction coatings. ${ }^{1,2}$ Even at the monolayer level, adsorbates can have a dramatic effect by reducing (as in the case of lubricants) ${ }^{3,4}$ or sometimes enhancing friction. ${ }^{5}$

The role of adsorbates and the generation of third bodies from ruptured chemical bonds at small scales is clearly worthy of experimental study, especially with atomic force microscopy (AFM) techniques, which have already provided fundamental insights into many aspects of friction and adhesion. ${ }^{6}$ While there have been many nanometer scale

a) Author to whom correspondence should be addressed; electronic mail: salmeron@stm.lbl.gov studies of friction in the elastic, wearless low-load regime, relatively few studies on that scale deal with the wear regime. Wear due to transfer of atoms has been observed by Park et al. ${ }^{7}$ for calcite crystals in solution, by Kopta et al. for muscovite mica in air, ${ }^{8}$ by Gnecco et al. for $\mathrm{KBr}$ in UHV, ${ }^{9}$ and by Helt et al. for muscovite mica in solution. ${ }^{10}$ These studies found that atomic scale wear can result from the enhanced chemical activity of the surface due to lowering of the activation barrier for reaction by the applied load as well as by electrochemical potentials.

On chemically active and highly adhesive substrates wear results in significant amounts of material being detached from one of the surfaces. In both cases, third bodies that can influence the friction behavior are generated. Qian et al. ${ }^{11}$ showed reproducible transient behavior in the friction properties for AFM tips scanning in contact with various surfaces, with several tens of scans being required before friction became reproducible. They proposed that the phenomenon is due to transfer of material between the tip and sample and observed that the mechanism of transfer depends on the relative humidity, applied load, and the material being scanned. Similarly, Carpick et al. ${ }^{12}$ observed that the frictional shear strength and interfacial adhesion energy of a $\mathrm{Pt} /$ muscovite mica interface in ultrahigh vacuum progressively decreased with each scan, but recovered if the tip was "cleaned" by blunting it to expose fresh Pt. The authors 

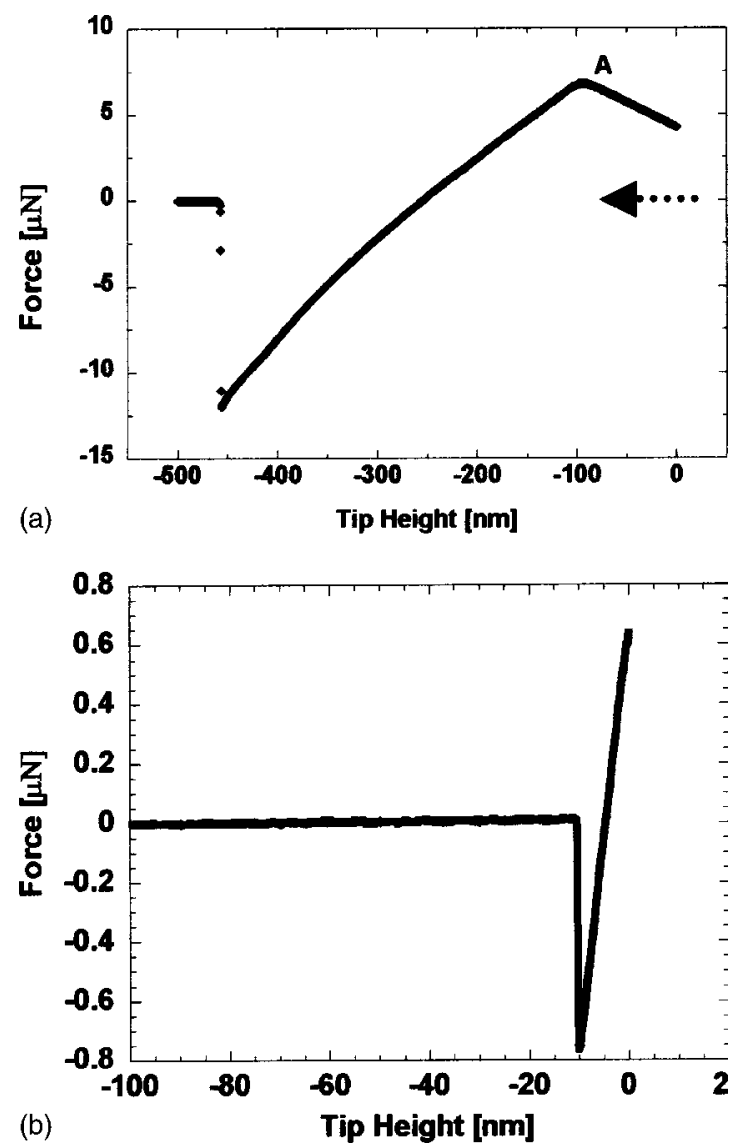

FIG. 1. (a) Force-displacement curve for a tungsten carbide tip in contact with a clean $\mathrm{Pt}(111)$ sample. The plot is shown for the retracting portion only. The load appears to increase when retraction starts, even though the tip-sample separation is increasing. This is due to the strong adhesion of the tip to the surface, which prevents sliding. Consequently, the tip pivots about the contact point. After that, the load decreases down to the pull-off point, as is usual in force-displacement curves. The deformations of the cantilever giving rise to this behavior are illustrated in Fig. 2. (b) A forcedisplacement plot for the same lever, but taken over a passivated area of the $\mathrm{Pt}(111)$ sample. The adhesion force is much lower, and the unusual behavior in the plot due to frictional forces is not present.

suggested that potassium atoms transferred from the mica surface to the tip could explain the observed progressive reduction of adhesion and friction. Drummond et al. ${ }^{13}$ performed experiments in which $\mathrm{WS}_{2}$ nanoparticles suspended in tetradecane fluid were compressed and sheared between the two mica sheets of the surface forces apparatus. They found that the particles formed a transfer film of nanometerscale thickness on the mica that reduced friction appreciably. These results highlight the complex but important role such behavior plays in friction processes at the nanometer scale, and demonstrate the need for further study in this nascent area.

In this article, we discuss the behavior of a single asperity interface composed of a $\mathrm{Pt}(111)$ sample and a carbidecoated AFM tip using a combination of UHV AFM and scanning tunneling microscopy (STM) techniques. Since both the Pt sample and the carbide tip are conductive, we were able to measure the local electrical conductance of the contact and the friction force simultaneously. ${ }^{14,15}$ In addition, the conducting tip allows STM operation, whereby high-resolution

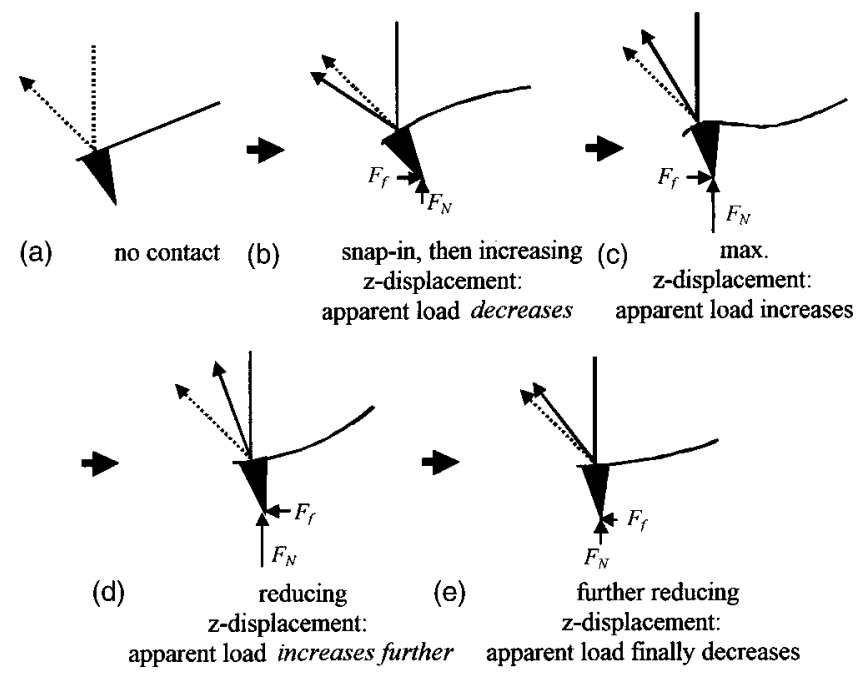

FIG. 2. Schematic drawing illustrating the deformation of a cantilever subject to high frictional forces. (a) The cantilever is shown at the upper left for the case of no load. The dashed line represents the trajectory of the laser for the zero load case, and is included in all subsequent sketches. (b) After snapping into contact, the $z$ displacement is increased. Normally, this would cause an increase in the laser signal (after the initial decrease due to the snap-in). However, large frictional forces cause bending at the end of the lever in the opposing direction, and producing an apparent decrease in the load. Friction is preventing the tip from sliding relative to the surface. (c) As the $z$ displacement is further increased, the frictional force eventually reaches its limiting value. The tip will begin to slide relative to the surface, and the upward bending induced by the $z$ displacement overtakes the tendency to bend in the opposite direction induced by the frictional force, so that now the apparent load begins to increase. Eventually the $z$ displacement reaches a maximum value. (d) As the $z$ displacement direction is reversed, the frictional force will now resist motion in the opposite direction, so that the bending it induces causes an apparent increase in the load. During this phase, the tip is not sliding relative to the surface. (e) Eventually the frictional force reaches a limiting value and once again the tip begins to slide. The apparent load will now decrease as the $z$ displacement is decreased. Stages (d) and (e) are clearly evident in Fig. 1(a).

noncontact images of the sample can be obtained before and after the contact experiments.

\section{EXPERIMENT}

The experiments were performed in a UHV chamber (base pressure $7 \times 10^{-11}$ Torr), equipped with a home-built $\mathrm{AFM}^{16}$ controlled by RHK electronics (RHK Technology, Troy, MI), low-energy electron diffraction (LEED), Auger electron spectroscopy (AES), differentially pumped ion sputtering, and sample cooling and annealing capabilities.

The Pt(111) single-crystal sample was cleaned by sputtering with $\mathrm{Ar}^{+}$ions of $1 \mathrm{keV}$ energy, both in hot conditions $\left(600^{\circ} \mathrm{C}\right.$ ) and at room temperature, for 10-20 min. After sputtering, $\mathrm{O}_{2}$ was introduced in the chamber at $10^{-6}$ Torr for $\sim 3$ min while the sample temperature was kept at $600^{\circ} \mathrm{C}$. Finally, the sample was flashed in vacuum to $950-1000{ }^{\circ} \mathrm{C}$ for $\sim 1 \mathrm{~min}$. The AES pattern taken after this procedure indicated a clean $\mathrm{Pt}(111)$ surface. A sharp (1 $\times 1)$ LEED pattern was also observed.

Commercial triangular silicon cantilevers with integrated tips, coated with approximately $20 \mathrm{~nm}$ of tungsten carbide ${ }^{16}$ were used for all measurements. The cantilevers were characterized by scanning electron microscopy in order to determine tip and lever dimensions, and also by AES to determine 

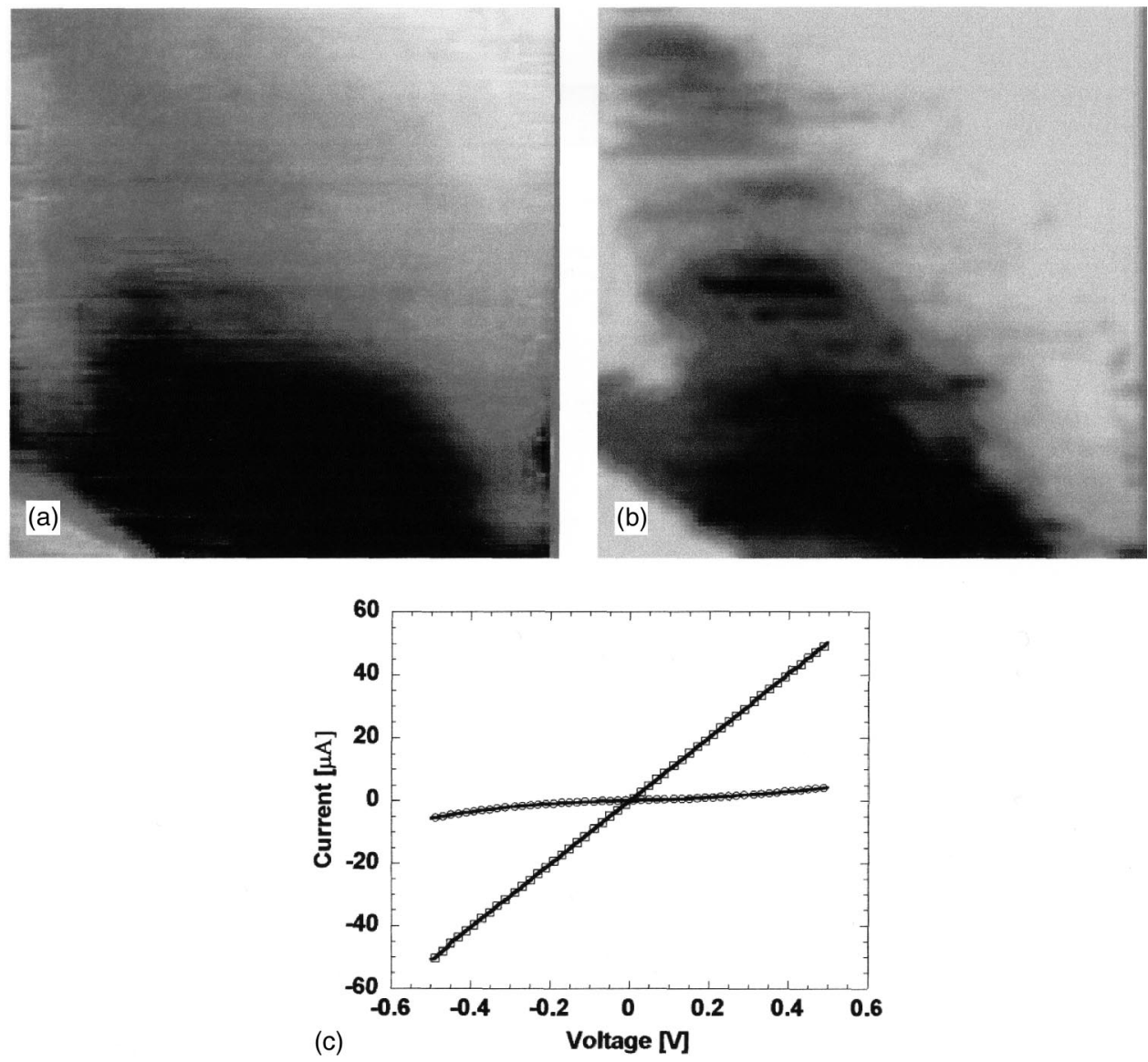

FIG. 3. Simultaneous friction (a) and point contact current (b) images of a $\mathrm{Pt}(111)$ surface acquired with a conductive WC-coated Si cantilever of 88 $\mathrm{N} / \mathrm{m}$ spring constant. Image size is $500 \times 500 \mathrm{~nm}^{2}$. Regions with high and low friction are clearly correlated to regions of high and low local conductivity. (c) Corresponding $I-V$ spectra acquired at the points of "clean" and "passivated" areas. The bias between tip and sample was varied from $-50 \mathrm{mV}$ to $+50 \mathrm{mV}$. High friction regions are correlated with ohmic conductance behavior, while the lower friction regions exhibit non-ohmic conductance, indicative of an insulating or semiconducting interlayer. the chemical composition of the lever and tip shaft. The measurements showed the presence of both tungsten oxide and carbide, which is not uncommon for such coatings. For convenience we will refer to these as "tungsten carbide" tips.

Two cantilevers with different spring constants of 88 and $3.1 \mathrm{~N} / \mathrm{m}$ were used. The stiffer cantilever was used for conductance and some friction measurements, while the other was used for the higher resolution friction measurements. The tips were treated in UHV prior to the measurements by applying short voltage pulses and/or by rubbing them against sacrificial areas of the surface. Normal cantilever force constants were taken from the manufacturer, and the normal/ lateral force ratio was calculated using the method described in Ref. 18. The absolute accuracy of the forces measured is limited due to significant uncertainty in the material properties of the cantilever and approximations used in the force constant calculations. However, relative changes in friction and adhesion could be accurately determined using the same cantilever probe during a series of measurements. A large dynamic range, two-stage $I-V$ converter was built, which provided low current gain (e.g., 10 ${ }^{4}$ ) for measurements of the current flowing through the tip-sample junction while in AFM-contact mode, and high gain (e.g., 109) for tunneling microscopy.

\section{RESULTS AND DISCUSSION}

\section{A. Irreversible adhesion between clean interfaces}

As mentioned above, the $\operatorname{Pt}(111)$ sample was cleaned by sputtering and annealing, and its state checked by AES and
LEED to verify the chemistry and structure of the surface. Because of the nanoscale dimensions of the tip apex, we could not assess spectroscopically its chemical state in the UHV chamber. However, we found that scanning at high loads on sacrificial areas of the sample consistently produced tips with highly adhesive properties and metallic conductance characteristics. The adhesion force for tips prepared in this manner was large enough that even at the lowest load, scanning was not possible without severe damage. These contacts were characterized by means of force-displacement curves, as in the example shown in Fig. 1(a), where a pull-off force of $L_{c}=12.0 \pm 1.2 \mu \mathrm{N}$ was measured with the cantilever of $88 \mathrm{~N} / \mathrm{m}$ normal spring constant. Assuming, for simplicity, an elastic adhesive contact, this force can be related to the work of adhesion of the interface. Within the extremes of the Johnson-Kendall-Roberts model for adhesive contacts, ${ }^{19}$ and the Derjaguin-Müller-Toporov model, ${ }^{20}$ we obtain an "effective" work of adhesion between 12 and $16 \mathrm{~J} / \mathrm{m}^{2}$. For this calculation, we used a value of $160 \pm 20 \mathrm{~nm}$ for the tip radius, which was measured experimentally by scanning over sharp edges of a faceted $\mathrm{SrTiO}_{3}(305)$ sample. ${ }^{21}$

This value of the interfacial work of adhesion is certainly an overestimation because we have neglected the contribution to the work of adhesion by plastic deformation in the junction. Nevertheless, the value is three orders of magnitude higher than that found in previous UHV measurements, ${ }^{6}$ such as $0.02 \mathrm{~J} / \mathrm{m}^{2}$ between silicon nitride tips and muscovite mica surface, or $0.4 \mathrm{~J} / \mathrm{m}^{2}$ for a Pt tip on mica. ${ }^{12}$ Note that the surface energy of most metals is in the 

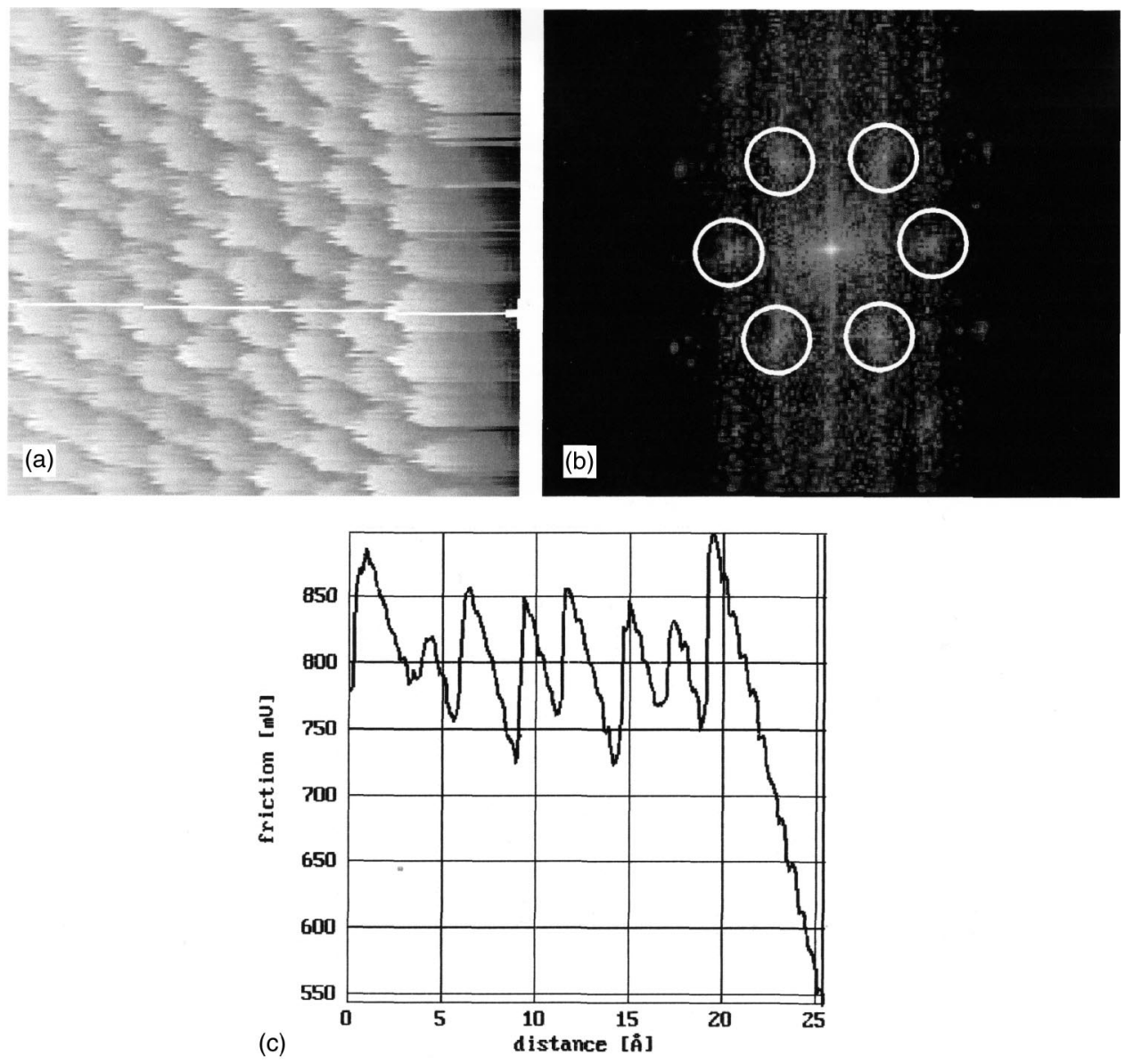

FIG. 4. (a) $2.5 \times 2.5 \mathrm{~nm}^{2}$ lateral force image obtained with a passivated tip and a clean $\mathrm{Pt}(111)$ sample. Atomiclattice stick-slip friction is clearly observed and provides proof that sliding is taking place along a clean Pt surface. (b) Fast Fourier transform of the data in (a), showing the periodicity and symmetry of the Pt(111) surface. (c) Line trace of the line indicated in (a), showing the clear stick-slip behavior. range of 1 to $5 \mathrm{~J} / \mathrm{m}^{2}$; that is, 2 to $10 \mathrm{~J} / \mathrm{m}^{2}$ are required to split an ideal crystal in half to create two new surfaces. ${ }^{22}$ Our value of $16 \mathrm{~J} / \mathrm{m}^{2}$ is beyond this range, consistent with the notion that the work of adhesion is overestimated by assuming an elastic contact. Nevertheless, the measurement indicates that strong bonds of several $\mathrm{eV}$ per atom are formed between the clean $\mathrm{Pt}(111)$ surface and the tungsten carbide tip. These bonds are also much stronger than those between silicon tips and clean $\mathrm{Cu}(111)$ reported by Bennewitz et al. ${ }^{23,24}$

A consequence of the strong adhesion is the unusual force-displacement plot observed when retracting the tip from the sample [Fig. 1(a) and Fig. 2]. The recorded data begins with the tip initially in contact, after being displaced approximately $450 \mathrm{~nm}$ making contact. Because of the strong bonding, the tip cannot slide over the surface and the cantilever is forced to adopt an S-shape like the one shown in Fig. 2(c). As the sample is retracted and the lever reverts to its normal bending shape [shown in Fig. 2(d)], it produces an apparent increase in the force initially. After passing through a maximum [point A in Fig. 1(a)], the force decreases as expected. This effect is explained by the strong friction force on a cantilever fixed at one end and with a tilted geometry with respect to the plane of the sample (22.5 in this case). The slope of the force-displacement curve is inverted because static friction prevents the tip from sliding with respect to the surface. Instead the tip is pivoting about the contact point, and the slope of the end of the cantilever is increasing.
Eventually the tip pivots enough that the maximum static friction force is reached and the tip can slide relative to the surface. Stages (d) and (e) sketched in Fig. 2 are evident in Fig. 1(a).

\section{B. Contacts between fully passivated interfaces: friction and conductance measurements}

As we have seen with clean tips and clean surfaces, contact-mode measurements cannot be performed without severely disrupting the contact region. To perform contact experiments while avoiding strong modifications, the surfaces must be chemically passivated. This can be achieved intentionally or unintentionally by the presence of adsorbate layers. An interesting question is whether these layers must be present on each or on only one of the surfaces for substantial passivation. The latter case implies that the layers are attached strongly to one of the surfaces and interact only weakly with the other, such that the contact can shear at this weak interface. As we will show in this and the next section, it is indeed possible to have both situations, which we shall call passivation when layers of material are present on each contacting surface, and half-passivation, when one of the two surfaces remains clean, during and after friction scanning.

On the Pt surface, the most common contaminant after annealing in UHV is carbon, as verified with AES. On the WC tip, in addition to oxygen present as a tungsten oxide, adventitious hydrocarbon or graphitic carbon can also accu- 

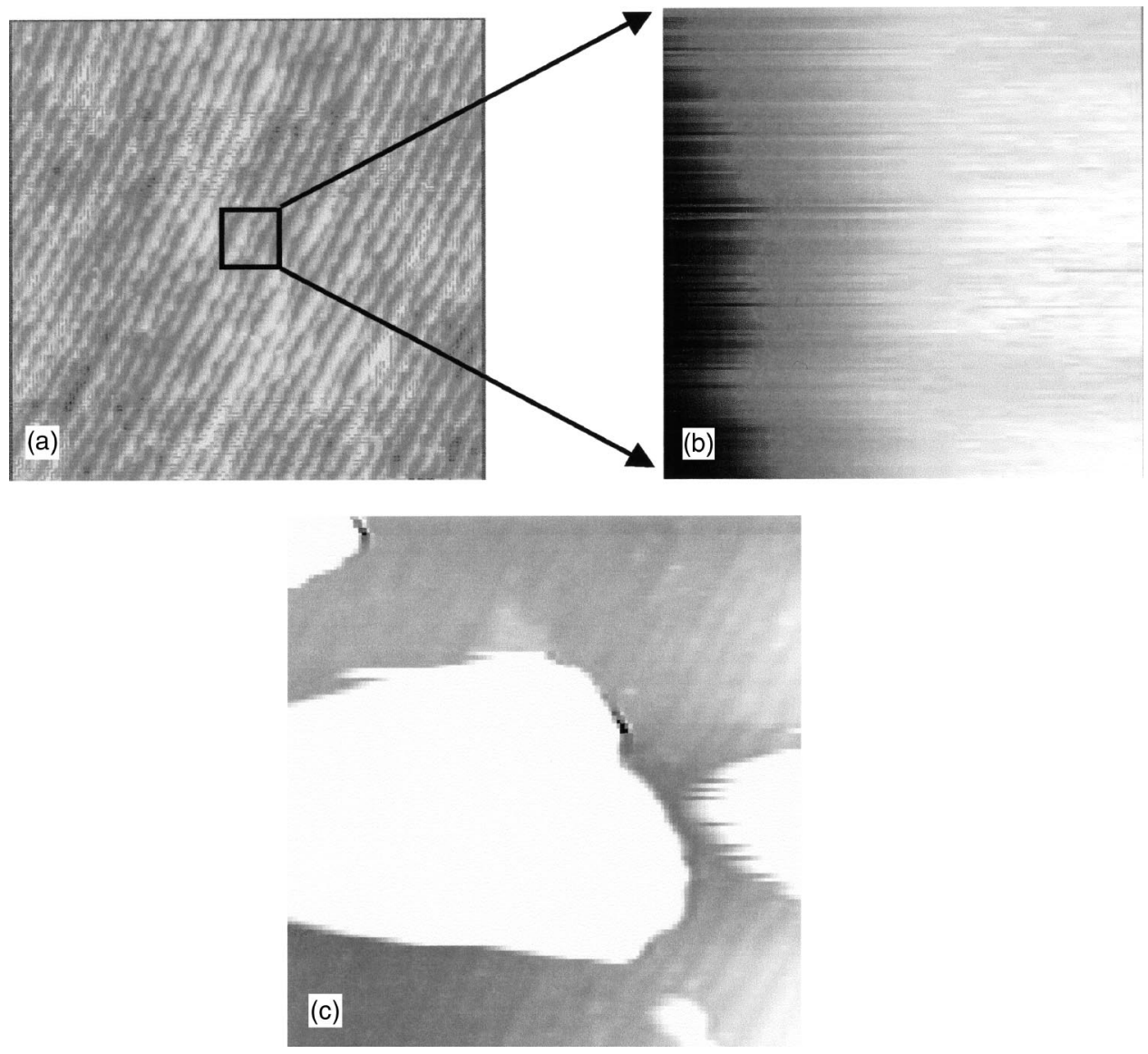

FIG. 5. (a) $100 \times 100 \mathrm{~nm}^{2}$ STM image of a highly stepped region of the $\operatorname{Pt}(111)$ surface. The image was acquired under the following tunneling conditions: bias $=-0.2 \mathrm{~V} ; \quad$ current $=200 \mathrm{pA}$. (b) $10 \times 10 \mathrm{~nm}^{2}$ AFM frictional force image acquired in contact at the center of (a). (c) Subsequent STM image, acquired under the same conditions as in (a), showing substantial material deposition from the AFM tip during the previous contact. mulate. Ex situ AES on the body of the cantilever did indeed reveal the presence of $\mathrm{O}, \mathrm{C}$, and $\mathrm{W}$ as the only observable constituents. We will first examine results where the Pt surface is covered by a layer of C-contamination, the only impurity element detected in the Auger spectra. Forcedisplacement data obtained with such passivated surfaces show low adhesion values in the range of $\sim 1 \mathrm{~J} / \mathrm{m}^{2}$, depending on the spatial location of the tip over the surface, as shown in the example of Fig. 1(b).

Topography, friction, and point contact current (corresponding to contact conductance) were recorded simultaneously as the tip was scanned over the surface, ${ }^{25}$ as shown in Fig. 3. The friction and conductivity maps exhibit a strong correlation, with regions of high and low friction corresponding to regions of high and low electrical conductivity, respectively. We propose that high friction and high local conductivity are associated with "cleaner" interfaces, while low friction and low local conductivity correspond to regions covered with more interfacial adsorbates. The spatial distribution of friction and conductance values remained consistent throughout several images. This indicates that the tip was not changing during the image acquisition, but rather, different regions of the sample had different amounts of adsorbates present.

Typical $I-V$ characteristics obtained with contacts in areas with different degree of passivation are shown in the graphs of Fig. 3(c). Ohmic behavior curves (straight lines) were always observed in the regions exhibiting high friction, while semiconductor-like behavior (sigmoid shapes) were observed in low friction areas. This observation can be understood on the basis of the poor conductivity of the contamination layers present, which decreases as their quantity, and thus passivation capacity, increases.

\section{Contacts with half-passivated interfaces: Atomic lattice resolution images}

In contrast with the fully passivated interfaces, when the Pt surface is clean, we could frequently observe stick-slip behavior with the atomic-lattice periodicity of the $\mathrm{Pt}(111)$ substrate, as shown in the image of Fig. 4(a) and the trace of a friction line in Fig. 4(c). The Fourier transform of the image [Fig. 4(b)] shows more clearly the three-fold symmetry with $0.27 \mathrm{~nm}$ periodicity, in agreement with the lattice constant of $\mathrm{Pt}(111)$. The image was acquired under zero externally applied load. The occurrence of stick-slip behavior was always associated with the presence of low adhesion, low friction, and low contact current, indicative of a chemically inactive tip.

This result indicates that the passivating layers are on the tip side of the interface, where they are bound strongly enough to withstand the applied shear stresses without transferring to the Pt surface. An analysis of the energy balance during friction is of interest here. Since the friction force is approximately $190 \mathrm{nN}$, the energy dissipated after a displacement of one unit cell $(\sim 3 \AA)$ is about $350 \mathrm{eV}$. Given the tip radius of $160 \mathrm{~nm}$, and a total load of $\sim 0.8 \mu \mathrm{N}$ [see Fig. 1(b)] a contact area of roughly $100 \mathrm{~nm}^{2}$ can be calculated contain- 
ing approximately 1000 atoms. This corresponds to an average energy dissipated per atom of $0.3 \mathrm{eV}$. We would predict that this energy is not enough to break the strong chemical bonds of the Pt atoms, and indeed that is what we observe.

\section{Tunneling experiments using the AFM tip}

Clean surfaces, and the propensity of the tip to transfer material to such surfaces, could be studied by taking advantage of the conductive nature of the AFM tip. This allows us to alternate between contact mode, and noncontact STM mode. The only requirement for STM operation is that the cantilever be sufficiently stiff to avoid the jump-to-contact instability ${ }^{6,17}$ at very small spatial gaps, i.e., $0.7-1.0 \mathrm{~nm}$ which is necessary for tunneling. We found that an $88 \mathrm{~N} / \mathrm{m}$ lever was sufficiently stiff to avoid such a jump-to-contact.

Figure 5(a) shows a $100 \mathrm{~nm} \times 100 \mathrm{~nm}$ STM image acquired at a bias of $-0.2 \mathrm{~V}$ and $200 \mathrm{pA}$ current. It shows a region of the $\mathrm{Pt}(111)$ with monoatomic steps separating terraces approximately $4 \mathrm{~nm}$ wide. Figure 5(b) shows a $10 \times 10 \mathrm{~nm}^{2}$ AFM contact friction force image at zero externally applied load acquired in the center of the previous image. During contact imaging the bias was held at $0 \mathrm{~V}$. The dark area on the left corresponds to the stick region, with slip occurring at approximately $1 / 3$ of the scan range. After withdrawing the tip from the contact, another STM image was acquired, as shown in Fig. 5(c), under identical tunneling conditions as in 5(a). It shows that material deposition took place with the interfacial rupture occurring inside the tip material.

Using the frictional force during a slip of several $\mu \mathrm{N}$, the energy dissipated during imaging can be estimated to be of the order of $10^{8} \mathrm{eV}$. The amount of material left on the surface [Fig. 5(c)] occupies an area of roughly $2500 \mathrm{~nm}^{2}$. The energy dissipated during friction is therefore sufficient to break the junction, even if very strong $(\sim 5 \mathrm{eV})$ bonds need to be broken.

\section{CONCLUSIONS}

By using AFM and STM with the same conductive WCcoated cantilevers, we were able to study the tribological and electronic properties of nanocontacts and to correlate these properties with the degree of passivation of the interface. Contacts could be classified as clean, half-passivated, and fully passivated, depending on whether none, one, or both of the participating surfaces is covered with chemically inactive layers. While it would be desirable to obtain detailed information on the specific chemistry and structure of these contaminant species, no technique currently exists for obtaining such information at a confined nanoscale interface. Rather, we are restricted to rely on wide-scale AES measurements of the surfaces. Based on these measurements, we propose that the passivating materials for the WC tips consist mostly of strongly bound $\mathrm{O}$ and $\mathrm{C}$ species. On the tip, they could be removed by sliding contact under high load on the Pt substrate. In the case of Pt, the contaminants were $\mathrm{C}$ species.

The clean Pt(111) surface could be imaged in STM mode with cantilevers stiff enough to avoid the jump-to-contact instability. When such a surface is brought into contact with a clean tip, strong bonds are formed that cause rupture of the contact in the bulk part of the tip and/or substrate upon separation. Sliding is strongly impeded in this case and always leads to severe cantilever deformations and distorted forcedisplacement curves.

With passivated tips, low adhesion energy contacts $\left(\sim 1 \mathrm{~J} / \mathrm{m}^{2}\right)$ are formed. The friction properties of such contacts depend on whether additional adsorbate layers are also present on the Pt surface. Passivated areas of the surface give rise to low-friction and sigmoid-type $I-V$ characteristics, typical of poorly conductive or semiconducting materials. Clean Pt areas produce ohmic contact characteristics.

Clean Pt can be imaged in contact mode with passivated tips and gives rise to atomic lattice stick-slip friction with the $\mathrm{Pt}(111)$ lattice periodicity. Thus, chemically active metal surface has been imaged in UHV in contact revealing stick-slip with atomic lattice periodicity, and indicates that the passivating layer on the WC tip is bound strongly enough to the tip that material is not transferred to the active Pt even in conditions where substantial energy dissipation takes place during friction.

The results indicate that even in ultrahigh vacuum conditions, transfer of low-conductivity, passivating material can easily occur in nanoscale contacts. The presence of these species substantially effects friction and adhesion. These results are relevant to the understanding of transfer film formation and its influence on the structural evolution and tribology of interfaces, whose inelastic properties are only beginning to be probed and understood at the nanometer scale.

\section{ACKNOWLEDGMENTS}

This work was supported by the Director, Office of Energy Research, Office of Basic Energy Sciences, Materials Sciences Division, of the U.S. Department of Energy under contract number DE-AC03-76SF00098. One of the authors (R. W. C.) acknowledges support from the National Science Foundation, Grant number CMS0134571, and the Office of Basic Energy Sciences, Materials Sciences and Engineering Division, U.S. Department of Energy, Contract No. DE-FG02-02ER46016.

${ }^{1}$ I. L. Singer, in Fundamentals of Friction: Macroscopic and Microscopic Processes, edited by I. L. Singer and H. M. Pollock (Kluwer, Dordrecht, 1992), Vol. 220, p. 237.

${ }^{2}$ I. L. Singer, MRS Bull. 23, 37 (1998).

${ }^{3}$ C. F. Mcfadden and A. J. Gellman, Surf. Sci. 39, 287 (1997).

${ }^{4}$ J. S. Ko and A. J. Gellman, Langmuir 16, 8343 (2000).

${ }^{5}$ G. He, M. H. Muser, and M. O. Robbins, Science 284, 1650 (1999).

${ }^{6}$ R. W. Carpick and M. Salmeron, Chem. Rev. 97, 1163 (1997).

${ }^{7}$ N.-S. Park, M.-W. Kim, S. C. Langford, and J. T. Dickinson, Langmuir 12, 4599 (1996).

${ }^{8}$ S. Kopta and M. Salmeron, J. Chem. Phys. 113, 8249 (2000).

${ }^{9}$ E. Genecco, R. Bennefewitz, and E. Meyer, Phys. Rev. Lett. 88, 215501 (2002).

${ }^{10}$ J. M. Helt and J. D. Batteas, Mica Surfaces: Charge Nucleation and Wear, in Encyclopedia of Nanoscience and Nanotechnology, edited by J. A. Schwarz, C. Contescu, and K. Putyera (Marcel Dekker, New York, 2004), Vol. 3, pp. 1947-1966.

${ }^{11}$ L. M. Qian, X. D. Xiao, and S. Z. Wen, Langmuir 16, 662 (2000).

${ }^{12}$ R. W. Carpick et al., Langmuir 12, 3334 (1996).

${ }^{13}$ C. Drummond et al., Adv. Funct. Mater. 11, 348 (2001).

${ }^{14}$ M. Enachescu, R. J. A. van den Oetelaar, R. W. Carpick, D. F. Ogletree, C. 
F. J. Flipse, and M. Salmeron, Phys. Rev. Lett. 81, 1877 (1998).

${ }^{15}$ M. Enachescu, R. J. A. van den Oetelaar, R. W. Carpick, D. F. Ogletree, C.

F. J. Flipse, and M. Salmeron, Tribol. Lett. 7, 73 (1999).

${ }^{16}$ Q. Dai et al., Rev. Sci. Instrum. 66, 5266 (1995).

${ }^{17}$ NT-MDT Inc., Moscow, Russia.

${ }^{18}$ D. F. Ogletree, R. W. Carpick, and M. Salmeron, Rev. Sci. Instrum. 67, 3298 (1996).

${ }^{19}$ K. L. Johnson, K. Kendall, and A. D. Roberts, Proc. R. Soc. London, Ser. A 324, 301 (1971).
${ }^{20}$ B. V. Derjaguin, V. M. Muller, and Y. P. Toporov, J. Colloid Interface Sci. 53, 314 (1975).

${ }^{21}$ R. W. Carpick, N. Agrait, D. F. Ogletree, and M. Salmeron, J. Vac. Sci. Technol. B 14, 1289 (1996).

${ }^{22}$ J. N. Israelachvili, Intermolecular and Surface Forces, 2nd ed. (Academic, London, 1992).

${ }^{23}$ R. Bennewitz et al., Tribol. Lett. 10, 51 (2001).

${ }^{24}$ R. Bennewitz et al., Phys. Rev. B 60, R11301-4 (1999).

${ }^{25}$ M. Enachescu et al., Phys. Rev. B 60, 16913-19 (1999). 